\title{
Prediction of cerebrovascular/cardiovascular disease secondary to metabolic syndrome: Ultrasonographic measures of vasodilator response
}

\author{
Hiroki Tsuchida, MD², Kiyoaki Iwashita ${ }^{1)}$, Yoko Tsuchiya ${ }^{1)}$, Hiroyuki Suda, MD²), Tomoko Nishi' \\ Asami Maruyama ${ }^{3)}$ and Noboru Motoyanagi ${ }^{3)}$
}

\begin{abstract}
:
Background: End-stage renal failure is escalating due to the increased prevalence of renal damage (chronic kidney disease, CKD) secondary to arteriosclerosis resulting from metabolic syndrome (MetS). In congruence with the observed renal damage, the occurrence of cerebrovascular/cardiovascular disease (CCVD) affecting the patient's vital prognosis and quality of life also increases. Our previous study reported on surrogate markers utilized for the early diagnosis of CKD pathophysiologically based on MetS. In the present study, the vasodilator response is investigated for their potential diagnostic value in the early diagnosis of CCVD. Subjects and methods: The vasodilator responses (flow-mediated dilation [FMD] and nitroglycerin-induced dilation [NID]) to the right brachial artery were measured accordingly as efficient indicators to serve as surrogate markers for early diagnosis of CCVD in 208 Japanese males who are diagnosed with MetS. Before the analysis of FMD and NID data, the subjects were divided into groups according to the presence or absence of CCVD or the number of risk factors for MetS. Results: FMD values were generally extremely low and showed no significant differences between the two groups with respect to the presence of CCVD or the number of MetS risk factors. In contrast, NID values were generally low but the decrease in NID values was significant and was more marked in the group with CCVD and in those with MetS risk factors compared to their respective pairing groups. Conclusion: Although FMD values already showed a decrease at the time of MetS diagnosis, NID values showed a delayed decrease, and this decrease in NID values was accompanied by the onset of CCVD. These findings suggest that FMD (lowered values) may serve as a surrogate marker for the early prediction of CCVD due to arteriosclerosis and that NID (lowered values) may serve as a surrogate marker for the diagnosis of a progressive pathophysiological change in CCVD.
\end{abstract}

Key words:

Metabolic syndrome, Arteriosclerosis, Metabolic syndrome nephropathy, Cerebrovascular/cardiovascular disease, Ultrasonic vasodilator responses

\section{Introduction}

Metabolic syndrome (MetS) $)^{1)}$ causes organopathies through arteriosclerosis. Typical organopathies secondary to arteriosclerosis include chronic kidney disease (CKD) and cerebrovascular/cardiovascular disease $(\mathrm{CCVD})^{2,3}$. Diabetic nephropathy (DN) and benign nephrosclerosis (BNS) which accounts for a large percentage of CKD cases are referred to as diabetic kidney disease (DKD) $)^{4)}$ by extension. This causes confusion presumably due to the extensive involvement of recent lifestyle changes, aging, and various treatment effects, in addition to the lacking consideration for the pathophysiological background of DN and BNS. To clarify the misperception, we propose to consider the concept of "metabolic syndrome nephropathy (MetS-N) ${ }^{\text {s.-8. }}$. This concept comprises the renal damage in patients with a pathophysiological back-

1) Research Institute of Strategy on Chronic Diseases, Association of Health-care and Medication for Labor

2) Department of Diabetes and Endocrinology, Tokyo Women's Medical University Yachiyo Medical Center

3) Department of Radiology, Chiba Kensei Hospital

Corresponding author: Hiroki Tsuchida, M.D., h-tsuchida@min-iren-c.or.jp

Received: July 10, 2020, Accepted: June 4, 2021

Copyright (C) 2021 Japan Society for Vascular Failure 
ground of MetS. Although the diagnosis of DKD is currently established by a combination of albuminuria (albumin-creatinine ratio $[\mathrm{ACR}]$ ) and estimated glomerular filtration rate (eGFR), it has been reported that a combination of the renal interlobar arterial resistive index (RI) and estimated renal blood flow (eRBF) permits a more accurate early diagnosis of MetS-N than the conventional method ${ }^{9}$.

The objective of the present study was to examine whether flow-mediated dilatation (FMD) and nitroglycerininduced dilatation (NID) - vasodilator responses of the brachial artery, measured by ultrasonography, can serve as surrogate markers for predictors/determinants of CCVD which frequently occurs with the progression of CKD as the pathophysiological basis of the MetS.

\section{Methods}

\section{Subjects}

A total of 208 Japanese males (named as grand total [GT]) who met the definition of Japanese $\mathrm{MetS}^{1}$ and could contribute to a set of FMD and NID data during the 8-year period between November 7, 2011 and October 28, 2019 were studied accordingly.

The subjects were divided into four groups: $(i)$ those with CCVD (group A: G-A, n=51) and those without CCVD (group B: $\mathrm{G}-\mathrm{B}, \mathrm{n}=157$ ); and (ii) those with four MetS risk factors (group $\mathrm{C}$ : $\mathrm{G}-\mathrm{C}, \mathrm{n}=152$ ) and those with three MetS risk factors (group D: $\mathrm{G}-\mathrm{D}, \mathrm{n}=58$ ), according to the presence or absence of CCVD.

The number of patients with obesity and other MetS risk factors (defined risk factors for MetS includes obesity [requisite], elevated blood pressure, lipid abnormalities, and elevated blood glucose).

The specification of the MetS risk factors was made according to the criteria of obesity (BMI $\geq 25$ and/or visceral fat area $\left.[\mathrm{VFA}] \geq 100 \mathrm{~cm}^{2}\right)$, elevated blood pressure $(\geq 130$ $\mathrm{mmHg}$ in systolic blood pressure [BPS] and/or $\geq 85 \mathrm{~mm} \mathrm{Hg}$ in diastolic blood pressure [BPD]), lipid abnormalities (triglyceride [TG] $\geq 150 \mathrm{mg} / \mathrm{dL}$ and/or HDL cholesterol [HDLC] $<40 \mathrm{mg} / \mathrm{dL}$ ), elevated fasting glucose (HbA1c $\geq 6.2 \%$ and/ or fasting plasma glucose [FPG] $\geq 110 \mathrm{mg} / \mathrm{d}$ ), and the use of antihypertensive medication, lipid-lowering medication, or diabetic medication.

\section{Relationship between CKD and CCVD}

The study subjects (G-T) were evaluated for the incidence of CCVD after the CKD was classified, for its characterization, into six eGFR categories $\left(\mathrm{G}_{1}\right.$ to $\mathrm{G}_{5}$ with $\mathrm{G}_{3}$ split into $3 \mathrm{a}$ and ${ }_{3 b}$ ) according to renal function.

\section{Evaluation Parameters}

The data was analyzed (mean \pm standard deviation [SD]) according to 13 parameters which included the subject's age, MetS-related factors (BMI, BPS, BPD, TG, HDL-C, HbA1c, and FPG), LDL-C, renal function (creatinine $[\mathrm{Cr}]$, eGFR, and ACR), and uric acid (UA).

The age data were obtained at the time the vasodilator response examination was conducted while the other characteristic data adopted in G-T indicated the worst values obtained throughout the course of the study, and similar characteristic data adopted in G-A, G-B, G-C, and G-D indicated the values obtained immediately before the examination.

\section{Procedure for FMD/NID Measurements}

FMD and NID measurements were performed by the following procedures.

1) Pretreatment: Each subject had breakfast regularly at 8:00 a.m. They were allowed to take their respective prescribed medications, if any. Smoking was prohibited and minimal amount of water intakes were allowed until 2:00 p.m. In a favorable environment (silent and dark conditions at around $25^{\circ} \mathrm{C}$ with $50 \%$ relative humidity), the blood pressure was measured after the subject had rested in the supine position for 10 minutes.

2) FMD/NID measurement: UNEXEF38G (UNEX Corp., Aichi, Japan) the ultrasound equipment utilized for the vascular function study.

3) FMD measurement: After the resting diameter was measured at the right brachial artery, an inflatable cuff for blood pressure measurement was placed around the right arm and inflated to a systolic blood pressure plus $50 \mathrm{mmHg}$. After 5 minutes of cuff inflation for blood expulsion, blood flow was restored. The brachial artery diameter was measured for 2 minutes when the maximum brachial diameter was reached after blood flow restoration to evaluate the vasodilator response of the brachial artery (FMD value). FMD (\%) was calculated using the following equation: FMD $(\%)=($ maximum diameter - resting diameter $) /($ resting diameter $) \times 100$.

4) After the completion of FMD measurement, each subject was placed at bed rest for 15 minutes and nitroglycerin $(0.3 \mathrm{mg})$ was sprayed sublingually to measure the maximum brachial artery for a 5-minute vasodilator response for NID measurement (NID value). NID (\%) was calculated using the following equation: NID $(\%)=$ (maximum diameter resting diameter $) /($ resting diameter $) \times 100$.

5) Cutoff value setting: Although statistics on normal cutoff values for FMD and NID in healthy Japanese individuals have not been reported, they have recently been published as $7.1 \%$ for FMD and $15.6 \%$ for NID by Maruhashi et $\mathrm{al}^{10)}$. Taking these published data into consideration, we performed a comparison among G-T, G-A, and G-B and the comparison among G-T, G-C, and G-D. Incidentally, a cutoff value of $4 \%$, the original value reported by UNEX Corporation, was used for the comparison of the FMD data across subdivision groups.

CCVD is defined as an ischemic heart disease (angina pectoris and cardiac infarction) or cerebral stroke (cerebral hemorrhage and cerebral infarction) that occurred during the entire clinical follow-up period prior to baseline measure- 


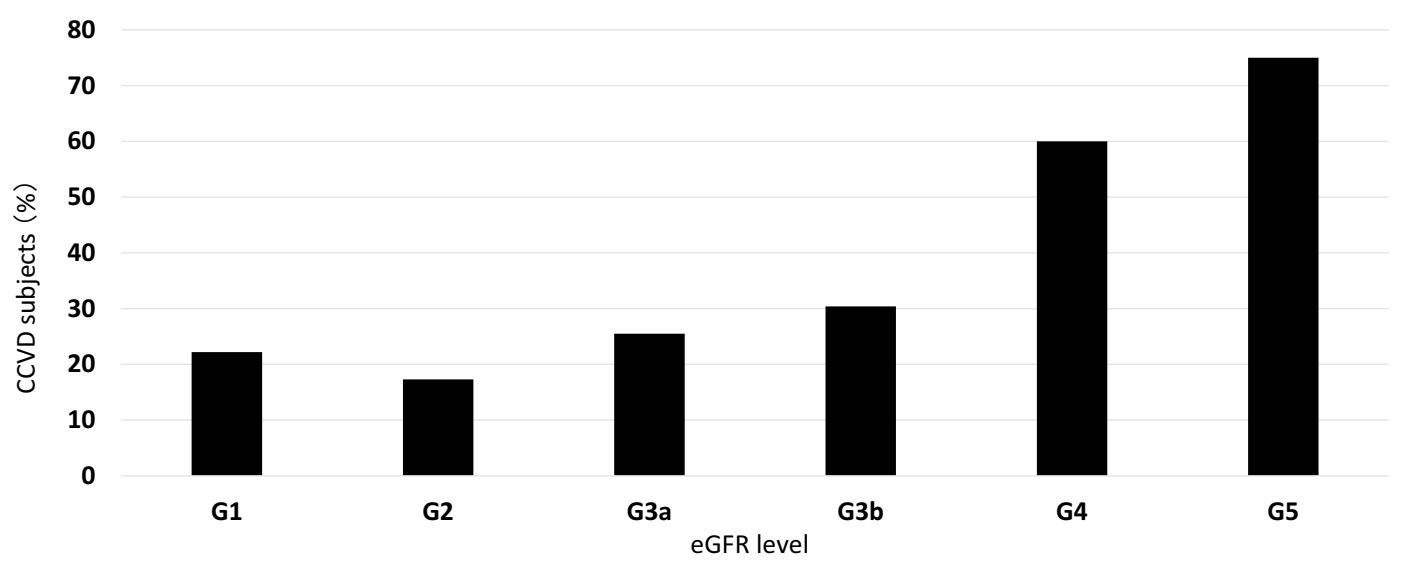

Figure 1. Percentage of subjects with CCVD by eGFR level Abbreviations: CCVD, cerebro-/cardiovascular disease; eGFR, estimated glomerular filtration rate.

Table 1. Baseline characteristics of study subjects by group

\begin{tabular}{lccccc}
\hline & $\begin{array}{c}\text { G-T } \\
\text { Total }\end{array}$ & $\begin{array}{c}\text { G-A } \\
\text { CCVD }(+)\end{array}$ & $\begin{array}{c}\text { G-B } \\
\text { CCVD }(-)\end{array}$ & $\begin{array}{c}\text { G-C } \\
\text { MetSRF=4 }\end{array}$ & $\begin{array}{c}\text { G-D } \\
\text { MetSRF=3 }\end{array}$ \\
\hline $\mathrm{N}$ & 208 & 51 & 157 & 152 & 56 \\
Age (yo) & $61.8 \pm 11.6$ & $67.5 \pm 9.5$ & $59.9 \pm 11.7$ & $61.5 \pm 11.6$ & $62.6 \pm 11.8$ \\
BMl (kg/m $\left.{ }^{2}\right)$ & $29.5 \pm 4.2$ & $26.2 \pm 4.1$ & $26.3 \pm 3.8$ & $26.5 \pm 4.1$ & $25.7 \pm 3.2$ \\
BPS $(\mathrm{mmHg})$ & $167.6 \pm 20.9$ & $142.7 \pm 16.8$ & $136.8 \pm 14.9$ & $139.8 \pm 15.3$ & $134.0 \pm 15.8$ \\
BPD (mmHg) & $101.3 \pm 11.8$ & $83.6 \pm 9.8$ & $82.1 \pm 9.2$ & $82.8 \pm 9.0$ & $81.7 \pm 10.5$ \\
TG (mg/dl) & $459.5 \pm 402.6$ & $168.5 \pm 102.1$ & $163.7 \pm 113.3$ & $174.7 \pm 114.6$ & $138.2 \pm 94.0$ \\
HDL-C (mg/dl) & $43.2 \pm 12.0$ & $55.6 \pm 15.0$ & $57.5 \pm 16.1$ & $56.2 \pm 15.3$ & $59.1 \pm 17.0$ \\
LDL-C (mg/dl) & $157.4 \pm 31.0$ & $116.8 \pm 30.4$ & $120.0 \pm 28.4$ & $119.6 \pm 29.6$ & $118.3 \pm 26.8$ \\
HbA1c (\%) & $8.4 \pm 2.6$ & $6.8 \pm 1.6$ & $6.5 \pm 1.0$ & $6.9 \pm 1.1$ & $5.7 \pm 0.5$ \\
FPG (mg/dl) & $196.9 \pm 89.4$ & $127.9 \pm 32.6$ & $128.0 \pm 36.3$ & $136.7 \pm 37.1$ & $105.9 \pm 16.1$ \\
UA (mg/dl) & $8.2 \pm 2.1$ & $6.2 \pm 1.5$ & $6.3 \pm 1.4$ & $6.3 \pm 1.5$ & $6.3 \pm 1.4$ \\
Cr (mg/dl) & $1.4 \pm 1.1$ & $1.4 \pm 1.0$ & $1.0 \pm 0.5$ & $1.1 \pm 0.7$ & $1.2 \pm 0.7$ \\
eGFR (ml/min/1.73m $\left.{ }^{2}\right)$ & $53.4 \pm 19.0$ & $56.1 \pm 25.8$ & $65.8 \pm 19.3$ & $64.6 \pm 22.1$ & $60.2 \pm 19.2$ \\
ACR (mg/gCr) & $346.3 \pm 887.6$ & $380.0 \pm 758.7$ & $168.4 \pm 586.2$ & $243.1 \pm 699.1$ & $144.1 \pm 391.0$ \\
\hline Da & &
\end{tabular}

Data are expressed as means \pm SD. Abbreviations: CCVD, cerebro-/cardiovascular disease; MetS, metabolic syndrome; MetSRF, MetS risk factors; G-T, group total; G-A, group with CCVD; G-B, group without CCVD; G-C, group with 4 MetSRF; G-D, group with 3 MetSRF; BMI, body mass index; BPS, systolic blood pressure; BPD, diastolic blood pressure; TG, triglyceride; HDL-C, high-density lipoprotein cholesterol; LDL-C, low-density lipoprotein cholesterol; HbA1c, glycated hemoglobin; FPG, fasting plasma glucose; UA, uric acid; $\mathrm{Cr}$, creatinine; eGFR, estimated glomerular filtration rate; ACR, albumin-creatinine ratio.

ment.

Statistical processing (non-paired $t$-test) was performed using the statistical analysis software Excel Statistics (BellCurve for Excel; Social Survey Research Information Co., Ltd., Tokyo, Japan). FMD and NID measurements and other tests were performed within the limits of the medical service fees (no consultation with the clinic's ethics committee was required). The chi-square test was used to examine the relationship between the renal function-based classification $\left(G_{1}\right.$ to $\mathrm{G}_{5}$ with $\mathrm{G}_{3}$ split into ${ }_{3 \mathrm{a}}$ and ${ }_{3 \mathrm{~b}}$ ) of $\mathrm{CKD}$ and the incidence of CCVD. Statistical significance was set at $p<0.05$.

\section{Results}

\section{Relationship between the Renal Function-based Classification of CKD and the Incidence of CCVD}

The incidence of CCVD increased with a corresponding decrease in eGFR, an index of renal function in CKD, as shown by $22.2 \%, 17.3 \%, 25.5 \%, 30.4 \%, 60.0 \%$, and $75.0 \%$ at eGFR levels of $\mathrm{G}_{1}, \mathrm{G}_{2}, \mathrm{G}_{3 \mathrm{a}}, \mathrm{G}_{3 \mathrm{~b}}, \mathrm{G}_{4}$, and $\mathrm{G}_{5}$, respectively (Figure 1).

\section{Laboratory Test Data}

Laboratory test data (means $\pm \mathrm{SD}$ ) from all the study subjects (G-T) and their subdivision groups (G-A, G-B, G-C, and G-D) are listed in Table 1. These baseline characteristic data were assessed for the significance of differences between G-A and G-B and between G-C and G-D. As shown 
Table 2. Assessment of the significance of intergroup difference

\begin{tabular}{lcccc}
\hline & G-A vs. G-B, $P$-value (two-sided) & significance & G-C vs. G-D, $P$-value (two sided) & significance \\
\hline Age & $<0.001$ & $* *$ & 0.549 & \\
BMI & 0.900 & & 0.114 & $*$ \\
BPS & 0.019 & $*$ & 0.016 & \\
BPD & 0.345 & & 0.460 & $*$ \\
TG & 0.786 & & 0.034 & \\
HDL-C & 0.460 & & 0.237 & $*$ \\
LDL-C & 0.498 & & 0.774 & $*$ \\
HbA1C & 0.147 & & $<0.001$ & \\
FPG & 0.978 & $*$ & $<0.001$ & \\
UA & 0.494 & $*$ & 0.876 & \\
Cr & 0.016 & & 0.626 & \\
eGFR & 0.016 & 0.189 & \\
ACR & 0.087 & & 0.215 & \\
\hline
\end{tabular}

Abbreviations: G-A, group with CCVD; G-B, group without CCVD; CCVD, cerebro-/cardiovascular disease; G-C, group with 4 metabolic syndrome risk factors; G-D, group with 3 metabolic syndrome risk factors; BMI, body mass index; BPS, systolic blood pressure; BPD, diastolic blood pressure; TG, triglyceride; HDL-C, high-density lipoprotein cholesterol; LDL-C, low-density lipoprotein cholesterol; HbA1c, glycated hemoglobin; FPG, fasting plasma glucose; UA, uric acid; $\mathrm{Cr}$, creatinine; eGFR, estimated glomerular filtration rate; ACR, albumin-creatinine ratio.

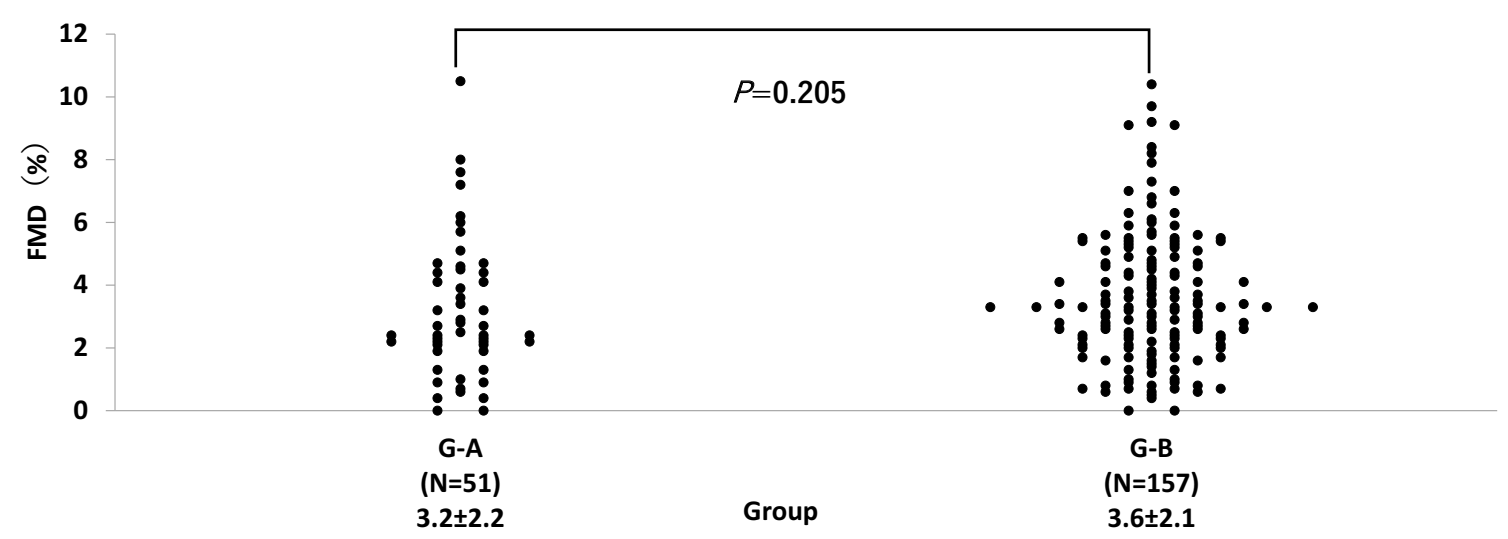

Figure 2. Presence or absence of CCVD and FMD

Abbreviations: CCVD, cerebro-/cardiovascular disease; FMD, flow-mediated dilatation

in Table 2, G-A had older age and higher BPS values than $\mathrm{G}-\mathrm{B}$. In addition, renal function ( $\mathrm{Cr}$ and eGFR) values were lower in group G-A than in group G-BB. When compared with G-D, G-C showed higher values of BPS, TG, HbA1c, and FPG.

\section{FMD Values (Comparisons between $G-A$ and $G-B$ and between G-C and G-D)}

The mean FMD value for G-T was $3.5 \pm 2.1 \%$ which was markedly lower than the $7.1 \%$, which is the established FMD cutoff value reported on healthy Japanese individuals. Of the total, 93.8\% (195 of 208 subjects) had FMD values lower than the FMD cutoff value. When $4.0 \%$, the FMD cutoff value reported by UNEX Corporation (Japan) was used as the reference standard, 63.9\% (133 of 208 subjects) had FMD values lower than the cutoff value. Furthermore, the mean FMD value was lower in G-A $(3.2 \pm 2.2)$ than in G-B $(3.6 \pm 2.1)$ as well as in G-C $(3.4 \pm 2.1)$ than in G-D $(3.8 \pm 2.2)$, although the differences between their respective pairing groups was not significant (Figures 2 and 3).

\section{NID Values (Comparisons between G-A and G-B and between G-C and G-D)}

The mean NID value for G-T was $13.3 \pm 5.9 \%$ which was lower than $15.6 \%$ which is the established NID cutoff value reported on healthy Japanese individuals. Of the total, 93.8\% (139 of 208 subjects) had NID values lower than the NID cutoff value. Furthermore, the mean NID value showed a significant difference between G-A (11.8 \pm 5.8$)$ and G-B $(13.8 \pm 5.9)$ and between G-C (12.8 $\pm 5.6 \%)$ and G-D (14.7 $\pm 6.3 \%)$. Additionally, statistical significance was lower in G-A than in G-B (=0.037) as well as in G-C than in G-D ( $p$ $=0.038)$ (Figures 4 and 5).

\section{Discussion}

The FMD/NID measurements were examined by ultrasonography to determine their functional utility as surrogate 


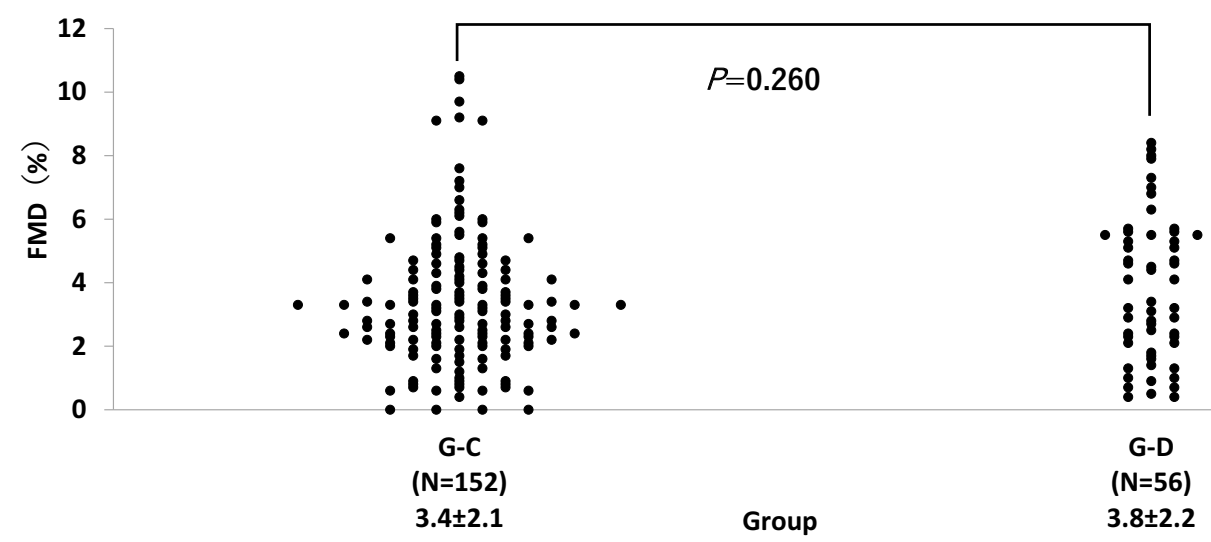

Figure 3. Number of MetS risk factors and FMD

Abbreviations: MetS, metabolic syndrome; FMD, flow-mediated dilatation

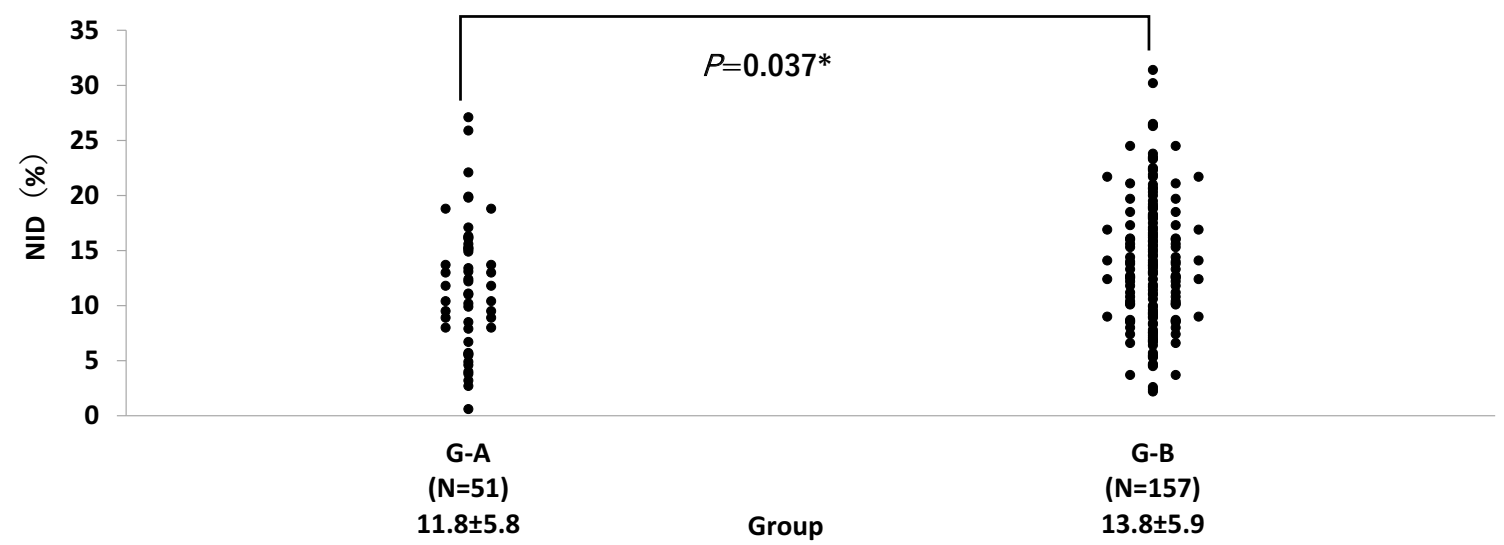

Figure 4. Presence or absence of CCVD and NID

Abbreviations: CCVD, cerebro-/cardiovascular disease; NID, nitroglycerin-induced dilatation

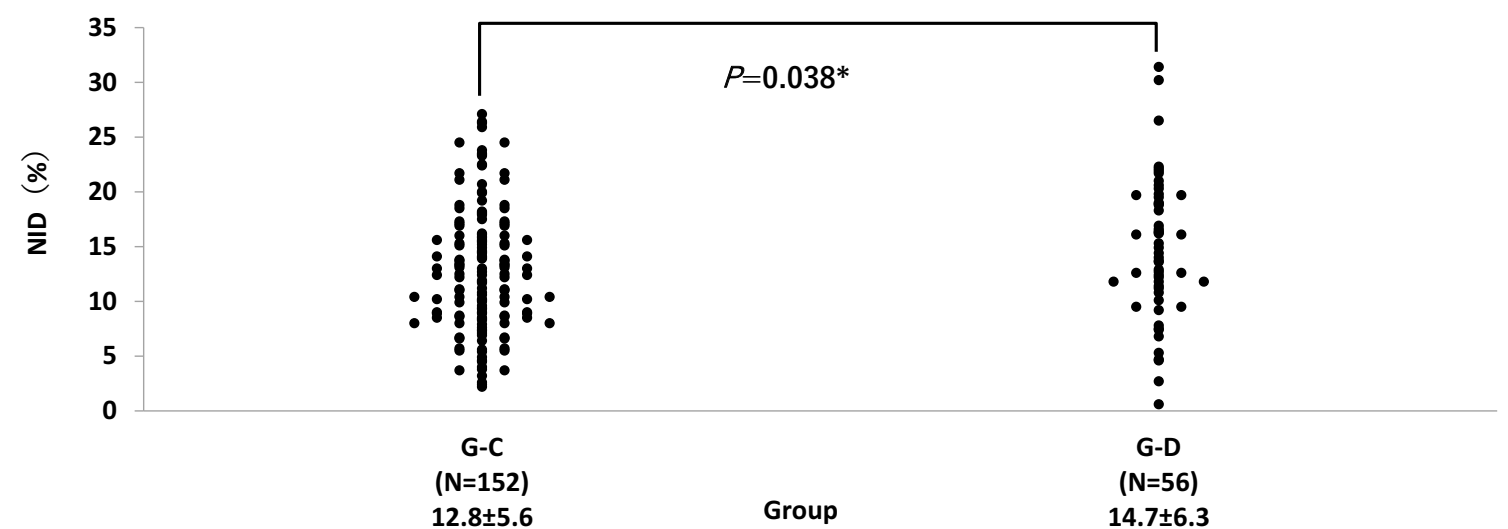

Figure 5. Number of MetS risk factors and NID

Abbreviations: MetS, metabolic syndrome; NID, nitroglycerin-induced dilatation

markers for the early prediction of CCVD which occurred frequently during the clinical follow-up period of $\mathrm{CKD}$ pathophysiologically based on MetS.

There were 208 subjects evaluated for the incidence of CCVD (Figure 1). The incidence of CCVD increased with a decrease in eGFR, an index of renal function in CKD, as shown by $22.2 \%, 17.3 \%, 25.5 \%, 30.4 \%, 60.0 \%$, and $75.0 \%$ at eGFR levels of $\mathrm{G}_{1}, \mathrm{G}_{2}, \mathrm{G}_{3 \mathrm{a}}, \mathrm{G}_{3 \mathrm{~b}}, \mathrm{G}_{4}$, and $\mathrm{G}_{5}$, respectively.
According to the results obtained, subjects with CCVD were already present at the eGFR level of $G_{1}$ to $G_{2}$, and the incidence of CCVD increased with worsening impairment of the renal function thereafter. These findings were reviewed for the effectiveness of FMD/NID as surrogate markers for the prediction of CCVD affecting the subject's vital prognosis and loss of quality of life. The results are discussed in the following sections. 


\section{FMD}

Maruhashi et al. reported that 7277 Japanese subjects had FMD values of $7.5 \% \pm 3.3 \%$ (median, $7.3 \%$; interquartile range, $5.3-9.4 \%$ ) in the no-risk group and $5.9 \pm 2.9 \%$ (median, 5.7\%; interquartile range, $3.9-7.5 \%$ ) in the risk group $^{10)}$. The mean FMD value for G-T was significantly lower than the FMD cutoff value. Furthermore, the mean FMD value was lower in G-A than in G-B and in G-C than in G-D, although the difference between the two subject groups were not significant (Figures 2 and 3). In other words, FMD had significantly lower values (as compared to the cutoff value) in general, without resulting in the detection of CCVD or increasing number of MetS risk factors. This finding suggests that, in the early stage of MetS, FMD values are already low and atherosclerotic lesions have already occurred.

\section{NID}

Maruhashi et al. ${ }^{10)}$ reported that 1764 Japanese subjects had NID values of $17.7 \pm 5.7 \%$ (median, $17.4 \%$; interquartile range, $13.2-21.7 \%$ ) in the no-risk group and $11.7 \pm$ $5.9 \%$ (median, $11.4 \%$; interquartile range, $7.4-13.5 \%$ ) in the risk group. The mean NID value for G-T was significantly lower than the NID cutoff value, in G-A than in G-B, and in $\mathrm{G}-\mathrm{C}$ than in G-D (Figures 4 and 5). These findings suggest that atherosclerotic lesions degenerate with an increasing number of MetS risk factors present, thereby causing CCVD.

FMD (an indicator of vascular endothelial cell dysfunction) and NID (an indicator of medial smooth muscle layer hypofunction) values were assessed in the target subjects diagnosed with MetS by taking into consideration the cutoff values (FMD, 7.1\%; NID, 15.6\%) defined in a previous study. At the time of assessment, abnormally low levels of FMD were observed in $93.8 \%$ of the subjects, whereas the levels of NID remained at $66.8 \%$. Incidentally, when the FMD cutoff value of $4.0 \%$ was used, low levels of FMD were found in $69.3 \%$ of the subjects. Meanwhile, when the NID cutoff value of $15.1 \%$ was used, low levels of NID were found in $60 \%$ of the subjects. If intimal and medial functions are assumed to be similar in the degree of deterioration, the adoption of an FMD cutoff value of $4.0 \%$ might be correct.

The FMD and NID data were then analyzed from the standpoint of the presence or absence of CCVD and the number of MetS risk factors. The FMD whose values were low in all target subjects showed no difference between the presence and absence of CCVD (G-A and G-B) or between 4 and 3 risk factors for MetS (G-C and G-D), whereas the NID whose values were not low in many subjects, showed a significant difference with regard to the presence or absence of CCVD and the number of MetS risk factors. These results suggest that the decrease in the FMD value in subjects with MetS might precede that of the NID value. This also suggests that FMD may be useful in arteriosclerosis-based diagnostic screening for CCVD while definitive diagnosis can be facilitated by NID.

These findings show that individuals diagnosed with MetS in their recent medical check-ups in Japan in 2008 should actively undergo ultrasonographic examination for RI/eRBF in the case of CKD and FMD/NID in the case of CCVD to consider for early diagnosis and proper treatment. This is expected to prevent the development and progression of these diseases.

\section{Conclusion}

With respect to the early diagnosis of CKD, it has already been reported that RI and eRBF, as measured by ultrasonography, are useful and important predictors of CKD pathophysiologically based on MetS.

Furthermore, confidently, in view of the findings that the decrease in the FMD value in target subjects indicated the onset of arteriosclerotic lesions and that the decrease in the NID value was associated with the progression of arteriosclerosis, FMD/NID measurements by ultrasonography demonstrates that it serves to be a useful significant surrogate marker for the early predictive/definite diagnosis of CCVD.

In summary, FMD and NID are important measurement parameters as predictors/determinants of CCVD, which occur frequently as the pathophysiological basis of MetS during the progression of renal damage leading to end-stage renal failure.

In conclusion, the findings suggest that the RI and eRBF measurements and FMD and NID measurements by ultrasonography may be utilized as an accurate diagnostic tool for the early diagnosis of CKD and CCVD as arteriosclerotic organopathies occurring on the basis of MetS. Thus, early comprehensive diagnosis for CKD and CCVD will produce a beneficial effect on their prevention and treatment.

\section{Acknowledgements}

We would like to thank Dr. Yasumi Uchida and Dr. Naotake Hashimoto for their careful guidance in writing the manuscript and to Mr. Toshio Hiroi for his kind assistance in translating it into English.

\section{Conflicts of Interest}

The authors have no financial conflicts of interest to disclose concerning this study.

\section{References}

1. Committee on Diagnostic Criteria for Metabolic Syndrome. Definition and diagnosis criteria of metabolic syndrome. J Jpn Soc Intern Med 2005; 94: 794-809.

2. Vanholder R, Van Biesen W, Verbeke F, Lameire N. The epidemic of cardio-vascular disease in renal failure: Where does it come from where do we go? Acta Clin Belg Sep-Oct 2006; 61(5): 20511.

3. Diagnosis, Prevention, and Treatment of Cardiovascular Diseases in People with Type 2 Diabetes and Pre-Diabetes: A Consensus 
Statement Jointed from The Japanese Circulation Society and The Japan Diabetes Society (ISBN978-4-524-22818-8). Tokyo: Nankodo Co., 2020 (in Japanese).

4. National Kidney Foundation. KDOQI Clinical Practice Guidelines and Clinical Practice Recommendations for Diabetes and Chronic Kidney Disease. Am J Kidney Dis 2007 Feb; 49(2 Suppl 2): S12S154.

5. Tsuchida H. TOPICS Metabolic syndrome nephropathy. Kidney and Dialysis 2006; 60(4): 638-40.

6. Tsuchida H. Preventive strategy for end-stage renal failure: A proposal of metabolic-syndrome-nephropathy on the point of highschool urinalysis in Chiba prefecture for 20 years. Chiba Med J 2012; 88: 11-25.

7. Iwashita K, Tsuchida H. Consideration of physiological examination and laboratory testing (Symposium). Rinsho Byori 2014; 62: 1104-9.
8. Tsuchida H. Metabolic syndrome nephropathy-Its clinicopathological features and early diagnosis. Kidney and Dialysis 2015; 78(4): 605-10.

9. Tsuchida H, Iwashita K. Metabolic syndrome nephropathy (earlydiagnosis of intrarenal arteriosclerotic lesions by renal ultrasonography). Vascular Failure 2018; 2(1): 45-52.

10. Maruhashi T, Kajikawa M, Kishimoto S, Hashimoto H, Takaeko Y, Yamaji T, et al. Diagnostic criteria of flow-mediated vasodilatation for normal endothelial function and nitroglycerin-induced vasodilatation for normal vascular smooth muscle function of the brachial artery. J Am Heart Assoc 2020 Jan 21; 9(2): e013915. doi: 10.1161/JAHA.119.013915.

11. Japanese Society for Dialysis Therapy Committee of Renal Data Registry. ILLUSTRATION Current status of chronic dialysis therapy in Japan (as of 31 December 2016). 2016. 